\title{
Crystallization of Amorphous Alumina Whiskers on Carbon Nanotubes Under Electron Beam Irradiation
}

\author{
Ahmed Jasim ${ }^{1}$, Xiaoqing He $\mathrm{He}^{2 \& 3}$, Tommi White ${ }^{2 \& 4}$ and Yangchuan Xing ${ }^{1}$ \\ 1. Department of Biomedical, Biological and Chemical Engineering, University of Missouri, Columbia, \\ MO, USA. \\ 2. Electron Microscopy Core, University of Missouri, Columbia, Columbia, MO, USA. \\ 3. Department of Mechanical \& Aerospace Engineering, University of Missouri, Columbia, MO, USA. \\ 4. Department of Biochemistry, University of Missouri, Columbia, MO, USA.
}

Aluminum oxide (alumina, $\mathrm{Al}_{2} \mathrm{O}_{3}$ ) is a highly nonconductive metal oxide with a band gap $~ 8.0 \mathrm{eV}[1]$. This oxide is widely used as an adsorbent, and a support for industrial catalysts in the oxidation of hydrocarbons. $\mathrm{Al}_{2} \mathrm{O}_{3}$ has many different structures as so-called polymorphs, such as $\beta, \gamma, \eta$, and $\theta$ phases. An amorphous phase $\left(\alpha-\mathrm{Al}_{2} \mathrm{O}_{3}\right)$ is the initial state for $\mathrm{Al}_{2} \mathrm{O}_{3}$ when it is synthesized either as a coating on various substrates or by itself. The amorphous phase transforms $\alpha-\mathrm{Al}_{2} \mathrm{O}_{3}$ to $\gamma-\mathrm{Al}_{2} \mathrm{O}_{3}$ at a high temperature. It has been reported that $\alpha-\mathrm{Al}_{2} \mathrm{O}_{3}$ becomes crystalline above $973 \mathrm{~K}$ in air [2]. Another way to create such transformation is by using irradiation with highly-energetic particles, such as ions, neutrons and electrons. Electron irradiation is one of the possible ways for structural control as an alternative to thermal annealing processes. A previous study reported a phase change from the $\alpha$ phase to $\gamma$ phase after irradiating by an electron beam in a range of acceleration voltages of $25-300 \mathrm{kV} \mathrm{[3].}$ The beam irradiation mechanism is elastic interaction with the material in which either weak bonds are broken, or unstable bonds are rearranged. In addition, when $\mathrm{Al}_{2} \mathrm{O}_{3}$ was irradiated under an intense beam, various defects, such as point defects and dislocations, were observed, leading to transformation to a metastable phase, $\kappa-\mathrm{Al}_{2} \mathrm{O}_{3}[4]$.

In this work, trimethylaluminium (TMA) was hydrolyzed on the surface of the carbon nanotubes (CNTs). The reaction mechanism consists of two steps, hydrolysis and condensation. If the hydrolysis rate is too fast or too slow, both steps could overlap, leading to producing an amorphous structure with a whisker morphology. We observed that these alumina whiskers on carbon nanotubes deformed, rearranged and crystalized, after they were irradiated by the electron beam. We investigated the surface topography by HR-TEM, electron energy loss spectroscopy (EELS), and energy filtered TEM elemental mapping. During the HR-TEM imaging, we observed a change in the $\mathrm{Al}_{2} \mathrm{O}_{3}$ layer morphology. The layer was distorted, and some diffraction spots showed up in the FFT patterns. The time for electron beam exposure was less than a minute with an acceleration voltage of $300 \mathrm{kV}$. Figure 1a shows TEM image of the $\mathrm{Al}_{2} \mathrm{O}_{3}$ on CNTs with a whisker alumina topography. Figure $1 \mathrm{~b}$-e show the elemental mapping along with an EELS spectrum taken from the whiskers showing only oxygen and aluminum in the whiskers. Figure 2 shows the HR-TEM images before and after electron beam irradiation inside the white-dotted circle. The FFT image shows clearly that the structure crystallized after the beam exposure. The lattice interplanar distance corresponding to the spots denoted by the red circle was indexed at 0.22 $\mathrm{nm}$, which indicates that a $\kappa-\mathrm{Al}_{2} \mathrm{O}_{3}$ phase was formed [5]. Further exploration of the exact conditions and mechanisms of this metastable phase $\kappa-\mathrm{Al}_{2} \mathrm{O}_{3}$ transformation will be detailed at the conference [6].

References:

[1] M Yazdanmehr et al., 7 (2012), p. 488. 
[2] M Tane et al., 59 (2011), p. 4631.

[3] R Nakamura et al., 113 (2013), p. 064312.

[4] C Chen et al., 63 (2010), p. 1013.

[5] B Holm et al., 59 (1999), p. 12777.

[6] This work was partially supported by an "Excellence in Electron Microscopy" award from the Electron Microscopy Core, University of Missouri (Award Number: FY2018).
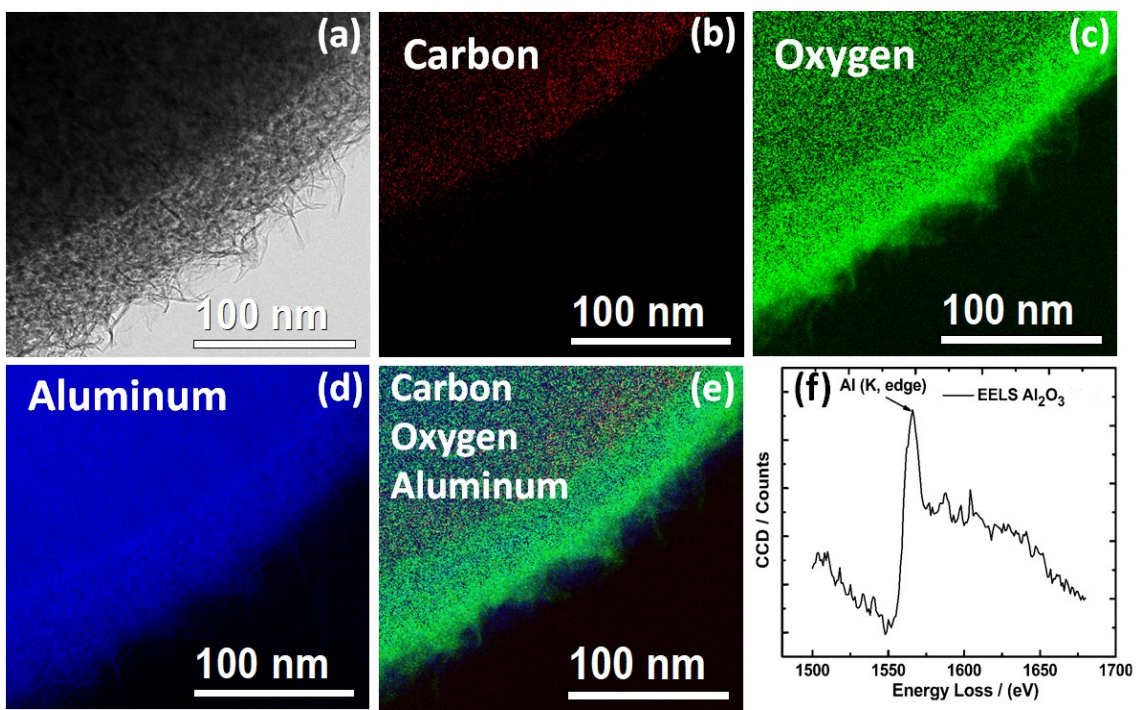

Figure 1. (a) TEM image of $\mathrm{Al}_{2} \mathrm{O}_{3} / \mathrm{CNT}$, (b-e) elemental mapping, (f) Electron energy loss spectrum.
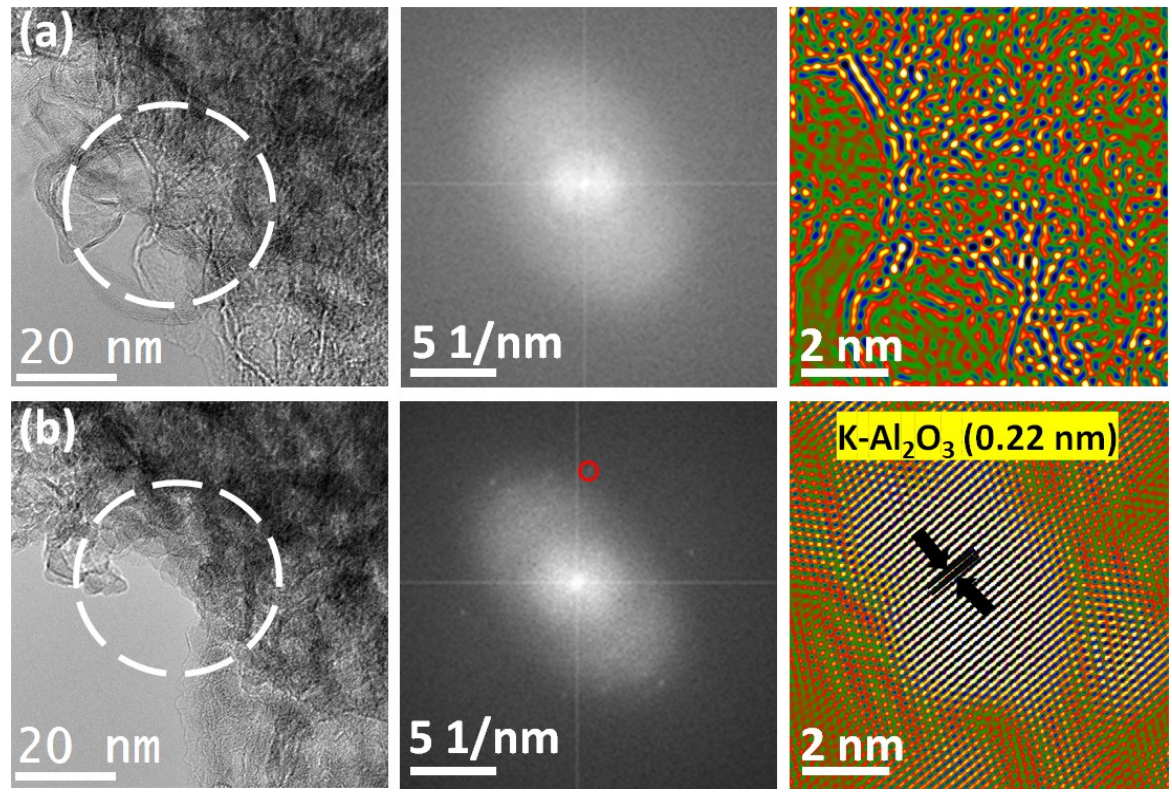

Figure 2. (a) Amorphous $\mathrm{Al}_{2} \mathrm{O}_{3} / \mathrm{CNT}$, HR-TEM with FFT, and inversed FFT on selected area, before beam irritation. (b) Semi-crystalline $\mathrm{Al}_{2} \mathrm{O}_{3} / \mathrm{CNT}$, HR-TEM with FFT (red circle) and inversed FFT on the selected area, after beam irritation. The white-dotted circle is the spot where the beam was focused. 